Pure and Applied Mathematics Quarterly

Volume 6, Number 4

(Special Issue: In honor of

Joseph J. Kohn, Part 2 of 2$)$

$1013-1025,2010$

\title{
Extremal Positive Pluriharmonic Functions on Euclidean Balls
}

\author{
Farhad Jafari* and Mihai Putinar ${ }^{\dagger}$ \\ Dedicated to Professor J.J.Kohn on the occasion of his seventy-fifth birthday
}

\begin{abstract}
Contrary to the well understood structure of positive harmonic functions in the unit disk, most of the properties of positive pluriharmonic functions in symmetric domains of $\mathbb{C}^{n}$, in particular the unit ball, remain mysterious. In particular, in spite of efforts spread over quite a few decades, no characterization of the extremal rays in the cone of positive pluriharmonic functions in the unit ball of $\mathbb{C}^{n}$ is known. We investigate this question by a geometric tomography technique, and provide some new classes of examples of such extremal functions.
\end{abstract}

Keywords: Pluriharmonic function, Riesz-Herglotz representation, extremal measure, Poisson transform

\section{INTRODUCTION.}

According to a classical theorem due to Herglotz and F. Riesz, every nonnegative harmonic function in the open unit disk $\mathbb{D}$ is the Poisson integral of a positive Borel measure on the unit circle $\mathbb{T}$. This correspondence readily characterizes the extreme points of the convex cone of positive harmonic functions $h$ in $\mathbb{D}$, normalized by the condition $h(0)=1$, as the Poisson integrals of extremal probability measures on the unit circle, namely the Dirac measures on the

Received November 27, 2007.

2000 Mathematics Subject Classification. 32U05, 31C10, 28A50

${ }^{*}$ Work partially supported by the IMA and the AFOSR

${ }^{\dagger}$ Work partially supported by the IMA and the National Science Foundation 
unit circle. Thus these extremal objects are simply the one-parameter class of functions

$$
u_{\zeta}(z)=P(z, \zeta), \quad \zeta \in \mathbb{T},
$$

where $P(z, \zeta)$ is the classical Poisson kernel in $\mathbb{D}$. This observation readily recasts the Riesz-Herglotz representation theorem as a Choquet integral: any positive harmonic function may be represented as a positively weighted integral of extreme points of the cone of positive harmonic functions in the disk.

Let $B^{2 n}$, or simply $B$ when there is no confusion, denote the open unit ball of $\mathbb{C}^{n}$ and $S^{2 n-1}$, or $S$, be the boundary of $B$. Recall that a $C^{2}$-function is pluriharmonic in $B$ if for every $a \in B$ and $b \in \mathbb{C}^{n} u_{a, b}(\lambda)=u(a+b \lambda)$ is harmonic for every $\lambda \in \mathbb{C}$ such that $a+b \lambda \in B$. Forelli [F1] has shown that if $u \in C^{\infty}(0)$ and $u_{\zeta}(\lambda)=u(\lambda \zeta)$ is harmonic in $\mathbb{D}$ for every $\zeta \in S$, then $u$ is pluriharmonic in $B$. Equivalently, $u$ is pluriharmonic in $B$ if and only if $u \circ \psi$ is harmonic in $B$ for every $\psi \in \operatorname{Aut}(B)$, where the latter denotes the group of complex analytic automorphisms of the ball. It is easy to see that Cayley's transform $f \rightarrow \frac{1+f}{1-f}=$ $F$ establishes a one-to-one correspondence between the positive pluriharmonic functions $u=\Re(F)$, normalized such that $F(0)=1$, and holomorphic maps $f: B^{2 n} \rightarrow B^{2}$ so that $f(0)=0$. An excellent reference to this topic is Rudin [R].

The aim of this note is to analyze the structure of the extremal points in the class of positive pluriharmonic functions on the unit ball of $\mathbb{C}^{n}$ and to produce some new examples of such functions. Establishing a Herglotz-Riesz representation theorem, via Choquet theory or not, valid for all positive pluriharmonic functions in $B$ is still a distant goal. This is due to the lack of an effective description of all extremal positive pluriharmonic functions.

Let $\mathrm{PH}_{0}^{+}(B)$ denote the class of positive pluriharmonic functions $u$ such that $u(0)=1$, and $\operatorname{Prob}^{h}(S)$ be the class of positive probability measures $\mu$ such that $u=P[\mu]$, where $P$ is the Poisson kernel of $B$. That is, $\operatorname{Prob}^{h}(S)$ is the set of all probablility measures on $S$ whose Poisson integral is pluriharmonic. Clearly, there is a one-to-one correspondence between $\mathrm{PH}_{0}^{+}(B)$ and $\operatorname{Prob}^{h}(S)$, and preimages of extremal points of $\mathrm{PH}_{0}^{+}(B)$ are extremal in $\operatorname{Prob}^{h}(S)$. Unfortunately this dictionary is of little use, as the class $\operatorname{Prob}^{h}(S)$ resists any intrinsic characterization. Since, $\operatorname{Prob}^{h}(S)$ is convex and weak* compact, the evaluation $\mu$ to $\mu(F)$ is upper semicontinuous on $\operatorname{Prob}^{h}(S)$, hence its maximum is attained at 
some extreme point of $\operatorname{Prob}^{h}(S)$. The maximal measures so obtained provide a sufficient condition for extremality. Whether all extreme measures are of this type would depend on strong convexity of the unit ball of $\operatorname{Prob}^{h}(S)$. If $f$ is an inner function on $B$, then $\Re(F)=\frac{1+f}{1-f}$ has zero radial limits a.e. on $\mathrm{S}$, and $F$ is generated by a singular measure in $\operatorname{Prob}^{h}(S)$. In particular, maximal singular measures, i.e. $\mathrm{PH}_{0}^{+}(B)$ functions generated by certain inner functions will be extreme. But not all inner functions necessarily generate an extremal $u$ in $\mathrm{PH}_{0}^{+}(B)$. The following result of Forelli shows that extreme points of $\mathrm{PH}_{0}^{+}(B)$ must have a special irreducibility property.

Theorem 1. ([F2]). If $u \in P H_{0}^{+}(B)$ is extremal and $u=\Re(F), F=\frac{1+f}{1-f}$, then $f$ is irreducible, i.e. there is no factorization of $f=g h$, where are holomorphic and $g, h: B^{2 n} \rightarrow B^{2}$.

Proof. To get a contradiction, assume $f$ is as above factorable. Without loss of generality, we can assume that $g(0)=0$ and $h(0)=i t, 0 \leq t \leq 1$. Put

$$
F_{1}=\frac{(1-g)(1-h)}{1-g h}+i t, F_{2}=\frac{(1+g)(1+h)}{1-g h}-i t .
$$

It is easy to see that $\Re F_{j} \geq 0$ and $F_{j}(0)=1$ for $j=1,2$. Since $F_{1}+F_{2}=2 F$, and $u=\Re(F)$ is extreme, $\Re\left(F_{1}\right)=\Re\left(F_{2}\right)$ and since $F_{j}(0)=1, F_{1}=F_{2}$. Hence

$$
h=i \frac{t+i g}{1+i t g} .
$$

Rewriting $F$ in terms of this $g$ and $h$, one gets

$$
F=\frac{1+t}{2} \frac{1+i g}{1-i g}+\frac{1-t}{2} \frac{1-i g}{1+i g}
$$

which implies that the real part of $F$ can be expressed as a convex combination of two distinct members of $P H_{0}^{+}(B)$, unless $t=1$. But then $h$ is a constant.

It is worth noting that Forelli's original theorem assumes $F$ is extremal. It is trivial to show that $u$ is extremal if and only if $F$ is extremal, since $F$ is uniquely determined by its real part $u$. A variant of another theorem of Forelli [F3] demonstrates an invariance property of the extreme points of $\mathrm{PH}_{0}^{+}(B)$.

Proposition 2. If $u$ is an extreme point of $\mathrm{PH}_{0}^{+}(B)$, and $\psi$ is an automorphism of $B$, then $v=\frac{u(\psi(z))}{u(\psi(0))}$ is an extreme point of $\mathrm{PH}_{0}^{+}(B)$. 
Proof. If $u \in P H_{0}^{+}(B)$, clearly $v \in P H_{0}^{+}(B)$ and $u \mapsto v$ maps $P H_{0}^{+}(B)$ onto $P H_{0}^{+}(B)$. Now suppose $u$ is an extreme point of $P H_{0}^{+}(B)$ but $v=t g+(1-t) h$ for $0 \leq t \leq 1$ and $g, h \in P H_{0}^{+}(B)$. Then the pre-image of $v$ in $P H_{0}^{+}(B)$ is a convex combination of pre-images of $g$ and $h$ with $s=\frac{\Re(t g(a))}{\Re(\operatorname{tg}(a)+(1-t) h(a))}, a=\psi^{-1}(0)$. Since $u$ is extreme, either $s=0$ or $s=1$, which imply either $t=0$ or $t=1$.

\section{Disintegration of Positive PluRiharmonic FunCtions}

By analogy to the disk, it is tempting to conjecture that, since a function $u$ is pluriharmonic if and only if each slice of $u$ is harmonic in the disk, and the extreme points of the positive harmonic functions are the Poisson kernels of Dirac measures on each slice, every positive pluriharmonic function on the ball is generated by these measures on the one-dimensional slices. Clearly, the automorphisms of $B$ leave this set invariant. Unfortunately, this intuition is false as it is shown by the following examples, also going back to Forelli [F4]

Example 3. If $n>1$, then $u=\Re(F), F=\frac{1+z_{1}^{2}+z_{2}^{2}+\cdots+z_{n}^{2}}{1-z_{1}^{2}-z_{2}^{2}-\cdots-z_{n}^{2}}$ is an extreme point of $\mathrm{PH}_{0}^{+}(B)$. Before we enter into the details of the instructive proof of this result presented in Section 3, note that $f(z)=z_{1}^{2}+z_{2}^{2}+\cdots+z_{n}^{2}$ is the Poisson kernel of the singular probability measure with mass of $\frac{1}{2}$ at $(1,0, \cdots, 0)$ and $(-1,0, \cdots, 0)$ and, by rotation invariance, along all great circles. Similarly, any monomial of the type, $f(z)=c z_{1}^{m_{1}} \cdots z_{n}^{m_{n}}$ with $c$ chosen such that $f(z)$ attains a maximum modulus 1 on $S$, and $\operatorname{gcd}\left(m_{1}, m_{2}, \cdots, m_{n}\right)=1$, and hence on a great circle through that point, will give boundary values which are Dirac measures which cannot be split.

On the basis of the above examples we are returning to general considerations, trying to better understand the structure of all positive pluriharmonic functions in the ball. Let $F \in \mathcal{O}(B), \Re(F)>0$ and $F(0)=1$. Taking boundary limits in the space of measures on the sphere

$$
w^{*}-\lim _{r \rightarrow 1} \Re F(r z) d \sigma(z)=d \nu(z)
$$

where $d \sigma$ is the normalized surface area measure on $S$. Hence one finds the representation

$$
F(z)=\int_{S}\left[\frac{2}{\left(1-w^{*} z\right)^{n}}-1\right] d \nu(w)
$$


Above, and throughout this article, we write $u^{*}=\left(\overline{u_{1}}, \ldots, \overline{u_{n}}\right)$, where $u=$ $\left(u_{1}, u_{2}, \ldots, u_{n}\right)$, and we adopt the notation $u^{*} v=\overline{u_{1}} v_{1}+\ldots+\overline{u_{n}} v_{n}$, for the Hermitian scalar product in $\mathbb{C}^{n}$

As noted by Koranyi-Pukansky [KP ] and the Russain school (Aizenberg, Dautov) $[\mathrm{AD}]$ not all positive measures on the sphere can arise as such boundary limits. They are restricted (and characterized) by the moment conditions:

$$
\left\{\begin{array}{lr}
\int_{S} u^{\alpha} \bar{u}^{\beta} d \nu(u)=0, & \alpha \not \leq \beta, \beta \not \leq \alpha \\
\int_{S} u^{\alpha} \bar{u}^{\alpha+\beta}\left[\alpha_{j}+\beta_{j}+1-(|\alpha|+|\beta|+n)\left|u_{j}\right|^{2}\right] d \nu(u)=0, & 1 \leq j \leq n \\
\int_{S} u^{\alpha+\beta} \bar{u}^{\alpha}\left[\alpha_{j}+\beta_{j}+1-(|\alpha|+|\beta|+n)\left|u_{j}\right|^{2}\right] d \nu(u)=0, & 1 \leq j \leq n .
\end{array}\right.
$$

The first line above comes from the fact that there are no mixed terms in $\Re F(r u)$, and the second and third from comparing the integral $\int_{S}\left|u^{\alpha+\beta}\right|^{2} d \nu(u)$ with $\int_{S}\left|u^{\alpha+\beta} u_{j}\right|^{2} d \nu(u)$.We will call a positive measure on $S$ that satisfies (1) a Korányi-Pukansky measure.

Restricting $F$ to complex lines, we obtain for each $\zeta \in S$,

$$
F(\lambda \zeta)=\int_{\mathbb{T}} \frac{1+\bar{\alpha} \lambda}{1-\bar{\alpha} \lambda} d \mu_{\zeta}(\alpha)
$$

where $\mu$ is a positive probability measure supported on the unit torus $\mathbb{T}$.

Combining these two observations one arrives at a balayage identity between a Koranyi-Pukanski measure $\nu$ and a family of probability measures on the torus, $\mu_{\zeta}$, namely

$$
F(\lambda \zeta)=\int_{S}\left[\frac{2}{\left(1-\lambda w^{*} \zeta\right)^{n}}-1\right] d \nu(w)=\int_{\mathbb{T}} \frac{1+\bar{\alpha} \lambda}{1-\bar{\alpha} \lambda} d \mu_{\zeta}(\alpha), \quad|\zeta|=1,|\lambda|<1 .
$$

By passing to a more restrictive class of functions, let $F(z)$ be the FantappièHerglotz transform of a positive measure $d m$ supported on $S$, that is:

$$
F(z)=\int_{S} \frac{1+w^{*} z}{1-w^{*} z} d m(w)
$$

For a discussion and relevance of such Fantappiè-Herglotz transforms in the unit ball, see $[\mathrm{McP}]$. Consequently, as above, the measure $d \mu_{\zeta}$ is the balayage of the push forward (projection) of $m$ on the complex line through $\zeta \in S$. 
Clearly if $c$ is a unimodular constant and we rotate $\zeta$ by $c$, we remain on the same circle. Hence

$$
d \mu_{c \zeta}(\alpha)=d \mu_{\zeta}(\bar{c} \alpha)
$$

i.e. $\mu_{\zeta}$ is rotationally invariant on the great circles through $\zeta$. Furthermore, to study the dependence on $\zeta \in S$, note that since $F=\frac{1+f}{1-f}$, with $f(0)=0$ and $f: B^{2 n} \rightarrow B^{2}$ is holomorphic, $F(\lambda \zeta)=\frac{1+f(\lambda \zeta)}{1-f(\lambda \zeta)}$ which implies that the function $\zeta \mapsto \mu_{\zeta}$ extends to a smooth function from the ball to probability measures on the one dimensional torus. Trying to determine how these "slice measures" are related to the projections of the original measure (e.g. a projection-slice theorem relative to a particular transform), a characterization of the extremal elements will be more transparent.

We record a first observation related to the integration of the slice measures associated to a holomorphic function with positive real part in the ball. Let $\hat{\mu_{u}}(\lambda)$ be the Szegö transform of the projection of $m$ through the complex line through $u \in S$, namely

$$
\hat{\mu_{u}}(\lambda)=\int_{\mathbb{T}} \frac{1+\bar{\alpha} \lambda}{1-\bar{\alpha} \lambda} d \mu_{u}(\alpha) .
$$

The relationship between $\hat{\mu_{u}}$ and the Fantappié-Herglotz transform of $m, F$, is made explicit in the next two results.

Theorem 4. Let $\mu_{z}$ with $z \in B$ be a $C^{\infty}$ family of probability measures on $\mathbb{T}$, satisfying the automorphy condition

$$
\hat{\mu_{w u}}(\lambda)=\hat{\mu_{u}}(w \lambda) \quad \forall|w|<1,|u|<1 .
$$

Then the function

$$
F(\lambda u)=\int_{\mathbb{T}} \frac{1+\bar{\alpha} \lambda}{1-\bar{\alpha} \lambda} d \mu_{u}(\alpha), \quad|\lambda|=1,|u|<1
$$

is holomorphic in $B, \Re(F) \geq 0$ and $F(0)=1$. Furthermore, the family $\mu_{z},|z|<$ 1 , is uniquely determined by $\Re(F)$.

Proof. By its very definition, the function $F(\lambda u)$ is $C^{\infty}$ in both variables $|\lambda| \leq 1$ and $|u|<1$, plus, it is holomorphic on every complex line passing through the origin. Thus Forelli's theorem [F1] implies that $F$ is holomorphic with $\Re F(z)>0$. 
Moreover,

$$
d \mu_{z}(\zeta)=\Re F(\zeta z) \frac{d \zeta}{2 \pi i \zeta}, \quad|z|<1
$$

With the above dictionary between the class $\mathrm{PH}_{0}^{+}(B)$ and smooth, automorphic maps into probability measure on the torus:

$$
\mu: B^{2 n} \longrightarrow \operatorname{Prob}(\mathbb{T}),
$$

understood, we would like to push the correspondence to the boundary of the ball, to the well defined map, still denoted by the same symbol:

$$
\mu: S^{2 n-1} \longrightarrow \operatorname{Prob}(\mathbb{T}) \text {. }
$$

The above relation provides an explicit relation of the slice measures on the parameter $u \in S$. In particular, this dependence is smooth, rotationally invariant, and the moments of $\mu_{u}$ are

$$
\int_{\mathbb{T}} \lambda^{k} d \mu_{u}(\lambda)=\int_{\mathbb{T}} \lambda^{k} \Re F(\lambda u) \frac{d \lambda}{2 \pi i \lambda}, \quad k \in \mathbb{Z} .
$$

Let

$$
F(z)=\sum_{|\alpha|=0}^{\infty} c_{\alpha} z^{\alpha}
$$

be Taylor's expansion of the holomorphic function $F$ in the unit ball. Consequently we obtain

$$
\begin{aligned}
& \int_{\mathbb{T}} \lambda^{k} d \mu_{u}(\lambda)=\frac{1}{2} \sum_{|\alpha|=k} \overline{c_{\alpha}} u^{* \alpha}, \quad k>0, \\
& \int_{\mathbb{T}} \lambda^{-k} d \mu_{u}(\lambda)=\frac{1}{2} \sum_{|\alpha|=k} c_{\alpha} u^{\alpha}, \quad k>0,
\end{aligned}
$$

and

$$
\int_{\mathbb{T}} d \mu_{u}(\lambda)=1
$$

Thus, for a function $F$ with $\Re F \in P H_{0}^{+}(B)$ we can associate a map $u$ : $S^{2 n-1} \longrightarrow \operatorname{Prob}(\mathbb{T})$, with the property that for every trigonometric polynomial $p(\lambda, \bar{\lambda})$ we obtain a $C^{\infty}$ map defined on the sphere

$$
u \mapsto \int_{\mathbb{T}} p(\lambda, \bar{\lambda}) d \mu_{u}(\lambda) .
$$


As a matter of fact, the dependence of $\mu_{u}$ is smooth on a larger class of functions. For our specific aims, the following case is sufficient to consider.

Let $h(\lambda)$ be a real valued harmonic function defined in an open neighborhood $|\lambda|<R$ of the closed unit disk $(R>1)$. Write $h=\Re f$ with $f$ analytic in the disk of radius $R$, and choose $1<r<R$. Then Cauchy's Theorem implies, for $|\lambda|=1$ :

$$
\begin{aligned}
f(\lambda) & =\int_{|w|=1} \frac{f(r w)}{r w-\lambda} \cdot \frac{r d w}{2 \pi i} \\
& =\int_{|w|=1} \frac{f(r w)}{1-r^{-1} \lambda \bar{w}} \cdot \frac{d w}{2 \pi i w} \\
& =\frac{1}{2} \int_{|w|=1} f(r w)\left[\frac{1+r^{-1} \lambda \bar{w}}{1-r^{-1} \lambda w}+1\right] \frac{d w}{2 \pi i w} .
\end{aligned}
$$

In conclusion

$$
\begin{aligned}
\int_{\mathbb{T}} f(\lambda) d \mu_{u}(\lambda) & =\frac{1}{2} \int_{|w|=1} f(r w) \overline{F\left(r^{-1} w u\right)} \frac{d w}{2 \pi i w}+\frac{1}{2} f(0) \\
& =\int_{|w|=1} f(r w) \Re F\left(r^{-1} w u\right) \frac{d w}{2 \pi i w} .
\end{aligned}
$$

Therefore, the map $u \mapsto \int_{\mathbb{T}} h(\lambda) d \mu_{u}(\lambda)$ is smooth in $u$, as long as $h$ is a harmonic function defined in a neighborhood of the closed disk.

Quite remarkably, the last conclusion can be reversed, into the following statement.

Theorem 5. There exists a bijective correspondence between holomorphic functions $F(z)$ in the ball satisfying $\Re F(z)>0, F(0)=1$ and maps from the sphere into probability measures on the torus with the properties:

$$
\hat{\mu_{w u}}(\lambda)=\hat{\mu_{u}}(w \lambda) \quad \forall|w|<1,|u|<1 .
$$

and the map

$$
u \mapsto \int_{\mathbb{T}} h(\lambda) d \mu_{u}(\lambda)
$$

is $C^{\infty}$ for every harmonic function $h$ defined in a neighborhood of the closed unit disk.

We will resume in a separate work the analysis of the natural smoothness condition (in some distribution space sense) satisfied by the family of measures $\mu_{u},|u|=1$.

We can recast the above result in terms of the matrix realization of RieszHerglotz functions. Specifically, a probability measure on the torus is equivalent, via the spectral theorem, to a unitary operator with a prescribed cyclic vector of length one. 
Proposition 6. Every automorphic family of measures $\mu_{z}, z \in S^{2 n-1}$, as before defines a family of unitary operators $U_{z}, z \in S^{2 n-1}$, with a common cyclic vector $\xi$ (of length one) by the identity:

$$
<\left(w-U_{z}\right)^{-1} \xi, \xi>=\int_{\mathbb{T}} \frac{d \mu_{z}(\zeta)}{w-\zeta}, \quad|w|>1 .
$$

Moreover, the automorphy relation (4) reads as

$$
<\left(w-\lambda U_{u}\right)^{-1} \xi, \xi>=<\left(w-U_{\lambda^{*} u}\right)^{-1} \xi, \xi>|w|>1 .
$$

Again, we will expand separately this matricial realization of functions in the class $P H_{0}^{+}(B)$.

\section{Extremal FUnCtions}

Relation (3) establishes an injection from the extreme points of $\mathrm{PH}_{0}^{+}(B)$, via the slice measures, into smooth families of harmonic functions of a single complex variable. This can be interpreted as a balayage to the boundary: for $|u|=1$, the measure $d \mu_{\lambda^{*} u}$ is the balayage of the measure $d \mu_{u}$ restricted to the circle of radius $|\lambda|$. For $|\lambda|=1$, because we deal with measures on the same circle, no balayage is needed. Hence

$$
|\lambda|=1,|u| \leq 1 \Longrightarrow d \mu_{u}=\left(\lambda^{*}\right)_{*} d \mu_{\lambda^{*} u}
$$

where we have used ()$_{*}$ to denote the push forward operation on measures.

Example 7. Consider first the following simple rational function in $\mathbb{C}^{2}$

$$
f(z, w)=\frac{1+z}{1-z} .
$$

We compute the associated measure corresponding to a point $(a, b) \in S$, i.e. $|a|^{2}+|b|^{2}=1$. The case $a=1$ and $b=0$ stands aside and we have

$$
\mu_{(1,0)}=\delta_{(1,0)} .
$$

Choose another point $a \neq 1$ in which case

$$
\mu_{(a, b)}=\Re f\left(e^{i t}(a, b)\right) d t .
$$

When evaluated on harmonic functions on the unit disk this gives

$$
\int_{\mathbb{T}} h\left(e^{i t}\right) d \mu_{(a, b)}(t)=h(\bar{a}) .
$$


Thus, the measure lives on $\mathbb{T}$, but the above formula requires to solve the Dirichlet problem with boundary data $\left.h\right|_{\mathbb{T}}$ and evaluate the harmonic function $h$ at $a^{*}$. Indeed, this shows that the dependence $(a, b) \rightarrow \mu_{(a, b)}$ is smooth.

Now, if we consider

$$
F(z, w)=\frac{1+z^{2}+w^{2}}{1-z^{2}-w^{2}}
$$

and as above fix a point $(a, b)$ on the unit sphere and look at the measures on the slice passing through this point,

$$
\mu_{(a, b)}=\Re F\left(e^{i t} a, e^{i t} b\right) \frac{d t}{2 \pi},
$$

in the sense of boundary limits from inside the disk, in case singularities in the weight occur. For all harmonic functions $h(\zeta)$ continuous up to $|\zeta| \leq 1$,

$$
\int h d \mu_{(a, b)}=\frac{1}{2} h\left(-\sqrt{a^{* 2}+b^{* 2}}\right)+\frac{1}{2} h\left(\sqrt{a^{* 2}+b^{* 2}}\right) .
$$

This explains why the measure cannot be decomposed into a convex combination. When we extend to the interior of the ball, $(a, b) \in B$, the same formula has to be valid and taking only one summand, it will destroy the $C^{\infty}$ behavior at $(0,0)$. Magically, when we take them both, the singularity disappears because we deal with nice rational function

$$
\Re \frac{1+e^{i t}\left(a^{2}+b^{2}\right)}{1-e^{i t}\left(a^{2}+b^{2}\right)}
$$

These examples clearly demonstrate how to construct more general examples of measures which will not decompose as convex combinations. These measures must arise from nice rational functions of the form

$$
\Re \frac{1+e^{i t} f(z)}{1-e^{i t} f(z)}
$$

where each slice behaves nicely, but such that the decomposition exhibited in equation (4) loses its $C^{\infty}$ behaviour at 0 .

Variations on this theme abound. Forelli already describes another such family. Note that if $f(z, w)=2 z w$ is a monomial which takes the maximum modulus 1 on the boundary of the ball in $\mathbb{C}^{2}$, and $F(z, w)=\frac{1+2 z w}{1-2 z w}$, then at a point $(a, \bar{a})$ on the unit sphere, the measure on the slice passing through this point is

$$
\mu_{(a, \bar{a})}=\Re F\left(e^{i t} a, e^{i t} \bar{a}\right) \frac{d t}{2 \pi}=\Re \frac{1+2|a|^{2} e^{2 i t}}{1-2|a|^{2} e^{2 i t}} \frac{d t}{2 \pi}=\frac{1}{2} \delta_{-1}+\frac{1}{2} \delta_{1},
$$


whereas the measure through other slices through the origin are clearly not singular. Now, for all harmonic function $h(\zeta)$ continuous up to $|\zeta| \leq 1$,

$$
\int h d \mu_{(a, b)}=\frac{1}{2} h(-\sqrt{2 a b})+\frac{1}{2} h(\sqrt{2 a b}),
$$

which again, for the same reasons as noted above, fails to be decomposable into convex combinations. Indeed, if $\mu_{(a, b)}$ such decomposable so that each piece preserves the $C^{\infty}$ property at $\{0\}$, then by Forelli's theorem the two terms generated by the decomposition will be positive pluriharmonic and thus $F$ will not be extreme. To characterize all measures leading to this property, we need a family of measures for which the individual terms in the symmetric decompositions given by Eq. (5) are not smooth at the origin, but the sum will be smooth because of an appropriate cancellation.

Proposition 8. Let $t \in(0,1)$ and $\alpha: B^{2 n} \longrightarrow \overline{\mathbb{D}}$ be a function satisfying the automorphy condition (4) and such that, for any harmonic function $h$ on the closed disk, the map

$$
z \mapsto \int_{T} h d \mu_{z}:=\operatorname{th}(\alpha(z))+(1-t) h(-\alpha z),
$$

is $C^{\infty}$, but $t \delta_{\alpha(z)}-(1-t) \delta_{-\alpha z}$ fails to have this property.

Then the family of probability measures on the unit torus $\mu_{z},|z|<1$, is associated to an extremal element of $\mathrm{PH}_{0}^{+}(B)$.

Proof. If we let $f(z)=t h(\alpha(z))+(1-t) h(-\alpha(z))$, then $h(\alpha(z))=\frac{1}{t}(f(z)+g(z))$ and $h(-\alpha(z))=\frac{1}{1-t}(f(z)-g(z))$ will not be smooth at the origin, since $g$ is not smooth; however, if $f$ is smooth, then $\mu_{z}$ is smooth and thus, by Forelli's theorem, a generating measure for the positive pluriharmonic functions. Hence all such $\mu$ will be extremal.

It is not hard to see that Proposition 8 also gives a necessary condition for $\mu$ to be extremal. In analogy with the fact that every function has an even and odd decomposition, loosely speaking, if the odd component of these measures is not smooth, and the even one is smooth, that measure is extremal. Thus the set of extreme points of the class of positive pluriharmonic functions is quite large, and hard to describe explicitly.

The following corollary of a key result of Aleksandrov shows another facet of the intractable space $\operatorname{Prob}^{h}(S)$. 
Theorem 9. Let $\phi: S \rightarrow\left[0, \infty\left[\right.\right.$ be lower semi-continuous and $\|\phi\|_{1}=1$. Then there exists a positive Borel measure $\nu$ on $S$ such that $\nu \perp \sigma$ on $S$ and, letting $\Re(f(z))=P[\phi d \sigma-\nu](z), f(0)=0$ and $F(z)=\frac{1+f}{1-f}$ is positive pluriharmonic in $B$.

Proof. By Aleksandrov's $L^{1}$-modification theorem ([A1], [A2] or [R2], Theorem 10.1), if we set $g_{1}=0$ and $g_{2}(0)=0, g_{2} \not \equiv 0$, there exists a holomorphic $h$ on $B$ such that $P[\phi d \sigma]-\Re h$ is a nonnegative harmonic function in $B$ whose radial limits are 0 a.e. $[\sigma]$. In particular, there is a positive singular Borel measure $\nu$ so that $\Re(F(z))=P[\phi d \sigma-\nu]$ is pluriharmonic and, since $g_{2}(0)=0, h(0)=0$, $F(0)=1$.

It is easy to show that $u \rightarrow \mu_{u}$ is lower semi-continuous on $S$ and this map is an embedding of $S$ into a weak ${ }^{\star}$-compact subset of $\operatorname{Prob}(\mathbb{T})$. Hence the converse to the above theorem also holds. Thus, the boundary values of $\mathrm{PH}_{0}^{+}(B)$ are a.e. positive lower semi-continuous functions on $S$ and the image of $P H_{0}^{+}(B)$ into $\operatorname{Prob}^{h}(S)$ are those described by the above theorem.

\section{REFERENCES}

[A1] A.B. Aleksandrov. The existence of inner functions in the ball. Math. USSR-Sb. 46:143-159, 1983.

[A2] A.B. Aleksandrov. Function theory in the unit ball. in the vol. Several Complex Variables II (G.M. Henkin and A.G. Vitushkin, eds.), Springer, Berlin, 1994, pp 10\%-178.

[AMcC] J. Agler and J.E. McCarthy. Complete Nevanlinna-Pick kernels. J. Funct. Anal., 175(1):111-124, 2000.

[AD] L.A. Aizenberg and Sh.A. Dautov. Holomorphic functions of several complex variables with nonnegative real part. Traces of holomorphic and pluriharmonic functions on the Shilov boundary. Mat. USSR Sbornik, 99:342-355, 1976.

[APS] M. Andersson, M. Passare, and R. Sigurdsson. Complex Convexity and Analytic Functionals. Illustrated Edition, Birkhäuser, 2004.

[F1] F. Forelli. Pluriharmonicity in terms of harmonic slices. Math. Scand. 41:358-364, 1977.

[F2] F. Forelli, A necessary condition on the extreme points of a class of holomorphic functions. Pac. J. Math 73:81-86, 1977.

[F3] F. Forelli, Measures whose Poisson integrals are pluriharmonic II. Ill. J. Math. 19:584-592, 1975.

[F4] F. Forelli. Some extreme rays of the positive pluriharmonic functions. Can. J. Math., 31:9$16,1979$.

[Ko] A. Korányi. The Poisson integral for generalized half-planes and bounded symmetric domains. Ann. of Math., 82:332-350, 1965. 
[KP] A. Korányi and L. Pukansky. Holomorphic functions with positive real part on polycylinders. Trans. Amer. Math. Soc., 108:449-456, 1963.

$[\mathrm{McP}]$ J. McCarthy, M. Putinar. Positivity aspects of the Fantappiè transform. J. d'Analyse Math. 97:57-83, 2005.

[R] W. Rudin, Function Theory in the unit ball of $\mathbf{C}^{n}$. Springer-Verlag, Berlin, 1980.

[R2] W. Rudin, New Constructions of Function Holomorphic in the unit ball of $\mathbf{C}^{n}$. CBMS 63, American Mathematical Society, Providence, 1986.

[St] M. Stoll. Invariant Potential Theory on the unit ball of $\mathbf{C}^{n}$. Cambridge University Press, Cambridge, 1994.

Farhad Jafari

Department of Mathematics

University of Wyoming,

Laramie, WY 82071-3036

E-mail: fjafari@uwyo.edu

Mihai Putinar

Department of Mathematics

University of California at Santa Barbara,

California,93106-3080

E-mail: mputinar@math.ucsb.edu 